Physics

Physics Research Publications

Purdue University

Year 2005

An accretion-jet model for black hole
binaries: Interpreting the spectral and
timing features of XTE J1118+480

F. Yuan W. Cui

R. Narayan

This paper is posted at Purdue e-Pubs.

http://docs.lib.purdue.edu/physics_articles/254 


\title{
AN ACCRETION-JET MODEL FOR BLACK HOLE BINARIES: INTERPRETING THE SPECTRAL AND TIMING FEATURES OF XTE J1118+480
}

\author{
Feng Yuan and Wei Cui \\ Department of Physics, Purdue University, 525 Western Avenue, West Lafayette, IN 47907; \\ fyuan@physics.purdue.edu, cui@physics.purdue.edu \\ AND \\ RAMESH NARAYAN \\ Harvard-Smithsonian Center for Astrophysics, 60 Garden Street, Cambridge, MA 02138; narayan@cfa.harvard.edu \\ Received 2004 July 26; accepted 2004 November 3
}

\begin{abstract}
Multiwavelength observations of the black hole X-ray binary XTE J1118+480 have offered abundant spectral and timing information about the source and have thus provided serious challenges to theoretical models. We propose a coupled accretion-jet model to interpret the observations. We model the accretion flow as an outer standard thin accretion disk truncated at a transition radius by an inner hot accretion flow. The accretion flow accounts for the observed UV and X-ray emission, but it substantially underpredicts the radio and infrared fluxes, even after we allow for nonthermal electrons in the hot flow. We attribute the latter components to a jet. We model the jet emission by means of the internal shock scenario, which is widely employed for gamma-ray bursts. In our accretion-jet model of XTE J1118+480, the jet dominates the radio and infrared emission, the thin disk dominates the UV emission, and the hot flow produces most of the X-ray emission. The optical emission has contributions from all three components: jet, thin disk, and hot flow. The model qualitatively accounts for timing features, such as the intriguing positive and negative time lags between the optical and X-ray emission and the wavelengthdependent variability amplitude.
\end{abstract}

Subject headings: accretion, accretion disks - black hole physics - ISM: jets and outflows stars: individual (XTE J1118+480) — X-rays: stars

Online material: color figure

\section{INTRODUCTION}

Strong evidence now exists for black hole primaries in $15 \mathrm{X}$-ray novae (also known as soft X-ray transients; McClintock \& Remillard 2005). One such source, XTE J1118+480, was discovered with the all-sky monitor aboard the Rossi X-Ray Timing Explorer (RXTE) on 2000 March 29 (Remillard et al. 2000). Subsequent optical observations led to a measurement of the mass function, $f(M)=6.00 \pm 0.36 M_{\odot}$, which represents a lower limit on the mass of the compact primary and thus makes the source a secure black hole candidate (BHC; McClintock et al. 2001a; Wagner et al. 2001). XTE J1118+480 is one of the best observed BHCs. It lies at an unusually high Galactic latitude $\left(+62^{\circ}\right)$, close to the Lockman Hole region. The foreground absorption is extremely low [with $N_{\mathrm{H}} \sim(0.7-1.3) \times 10^{20} \mathrm{~cm}^{-2}$; Hynes et al. 2000; McClintock et al. 2001b], which allowed the detection of the source by the Extreme Ultraviolet Explorer (EUVE) satellite (Hynes et al. 2000). Simultaneous (or nearsimultaneous) observations were conducted, on multiple occasions, at radio, infrared, optical, UV, EUV, and X-ray wavelengths with state-of-the-art instruments (Hynes et al. 2000; McClintock et al. 2001b, 2003; Frontera et al. 2001; Chaty et al. 2003).

For clarity, we briefly summarize the main observational results here. These include two aspects: spectral and timing features. The most complete spectral energy distribution (SED) of XTE J1118+480 is shown in Figures 1 and 2. The radio data are from Fender et al. (2001), and the infrared to X-ray data, from McClintock et al. (2001b) (all the data are associated with "epoch 2," when the best simultaneous coverages were achieved; see Chaty et al. [2003] for a summary of all observations). The radio spectrum is well described by a power law of the form $F_{\nu} \propto \nu^{0.5}$. Such a spectrum is often thought to be typical of jet emission, although no jet has been directly imaged, down to a limit of $<65 D(\mathrm{kpc}) \mathrm{AU}$ (Fender et al. 2001), where $D$ is the distance to the source. Note that we do not include in Figures 1 and 2 an observational data point at $350 \mathrm{GHz}$ (Fender et al. 2001), because this measurement was not done simultaneously with the others. From IR to UV, the spectrum is flat, with the Hubble Space Telescope (HST) spectrum exhibiting emission lines. In addition, a Balmer jump is seen in absorption at $\nu \approx$ $10^{14.9} \mathrm{~Hz}$ (Hynes et al. 2000), implying that thermal emission contributes substantially to the optical/UV band. The derived EUV spectrum depends sensitively on the assumed $N_{\mathrm{H}}$, which is still not well constrained but probably lies in the range $N_{\mathrm{H}}=$ $(1.0-1.3) \times 10^{20} \mathrm{~cm}^{-2}$ (McClintock et al. 2001b, 2004). We take this uncertainty into account by requiring the model to stay within the allowed range at EUV energies. McClintock et al. (2001b) fitted the X-ray spectrum with a broken power law. Above $\sim 2 \mathrm{keV}$ they obtained a photon index of $\sim 1.78$, but below $\sim 2 \mathrm{keV}$ the spectrum appeared to be relatively harder. However, calibration issues were subsequently noted for the ACIS detectors used in the Chandra observations. ${ }^{1}$ This makes the spectrum uncertain at low energies. There is, in fact, independent evidence that the break at $2 \mathrm{keV}$ may not be real. XTE J1118+480 was observed many times with BeppoSAX, but the X-ray spectra show no apparent deviation from a single power law at low energies (Frontera et al. 2001).

\footnotetext{
${ }^{1}$ See http://cxc.harvard.edu/cal/Acis/Cal_prods/qeDeg/index.html.
} 


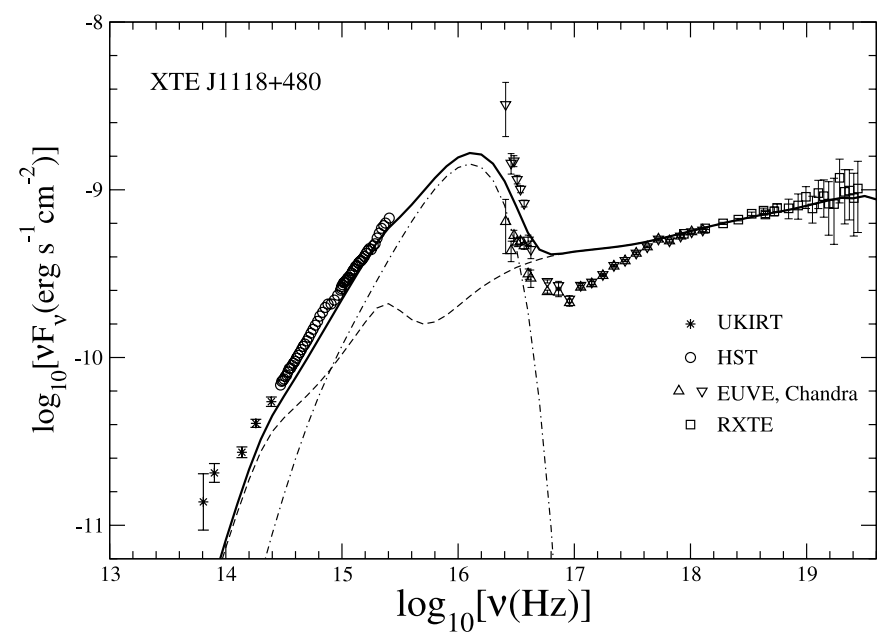

FIG. 1.-Spectral modeling results for XTE J1118+480. The fit was made with a model consisting of an inner hot accretion flow and an outer cool thin disk. The parameters of the model are $r_{\mathrm{tr}}=300 r_{\mathrm{S}}, \dot{M}_{0}=0.05 \dot{M}_{\mathrm{Edd}}, \alpha=0.3$, $\beta=0.9$, and $\delta=0.5$. The dashed line shows the emission from the inner hot accretion flow, the dot-dashed line shows the emission from the outer cool disk, and the solid line shows the sum of the two. The model explains the EUV and $\mathrm{X}$-ray data quite well, slightly underpredicts the optical/UV, and significantly underpredicts the IR and radio fluxes (the radio data are shown in Fig. 2). Note that two sets of EUV data are shown, for two different choices of $N_{\mathrm{H}}$. The X-ray spectral break at $\sim 10^{17.7} \mathrm{~Hz}$ may not be real (see text for details). [See the electronic edition of the Journal for a color version of this figure.]

The main timing features include the following. (1) A quasiperiodic oscillation (QPO) feature was detected in the X-ray light curve, initially at a frequency $\nu \sim 0.08 \mathrm{~Hz}$ (Revnivtsev et al. 2000), and was subsequently found to evolve (Wood et al. 2000). The QPO was also detected in the optical and UV bands at similar frequencies (Haswell et al. 2000). The fractional rms amplitude of the QPO is $8 \%-10 \%$ in X-rays but only about $1 \%$ at UV wavelengths (Hynes et al. 2003, hereafter H03). The fact that the same QPO frequency is seen at optical, UV, and X-ray wavelengths indicates a common origin. (2) XTE J1118+480 also shows rapid aperiodic variability at most wavelengths. The variability amplitude is quite large both in the X-ray and IR bands but is small in the optical/UV band. (3) Correlation between emission at different wavelengths is apparent (H03). In particular, cross-correlation analysis has revealed some puzzling details in the correlation between the optical and X-ray emission (Kanbach et al. 2001; H03; Malzac et al. 2003). In general, the optical photons appear to lag the X-ray photons by $1-2 \mathrm{~s}$ (see H03, although with caveats). The lags are wavelength dependent; on average a longer delay is seen at longer wavelengths. On the other hand, the cross-correlation function also shows a "precognition dip," i.e., the optical emission decreases about 2-5 s before the corresponding X-ray increase (Kanbach et al. 2001). At UV wavelengths the dip appears to be weaker, and the lag becomes shorter: $\sim 0.5 \mathrm{~s}$ (H03). These complicated positive and negative time lags between optical/UV and X-ray emission are not easy to understand. What is quite clear from the derived autocorrelation functions (ACFs) is that the optical/UV emission is not consistent with being due to the reprocessing of $\mathrm{X}$-ray photons by the accretion disk, as is often assumed, because the ACF at optical/UV wavelengths is narrower than that in X-rays (Kanbach et al. 2001; Spruit \& Kanbach 2002; H03).

Several models have been proposed to explain the observed spectral and temporal properties of XTE J1118+480. Esin et al. (2001, hereafter E01) explain the spectrum with an advectiondominated accretion flow (ADAF) model based on the work of

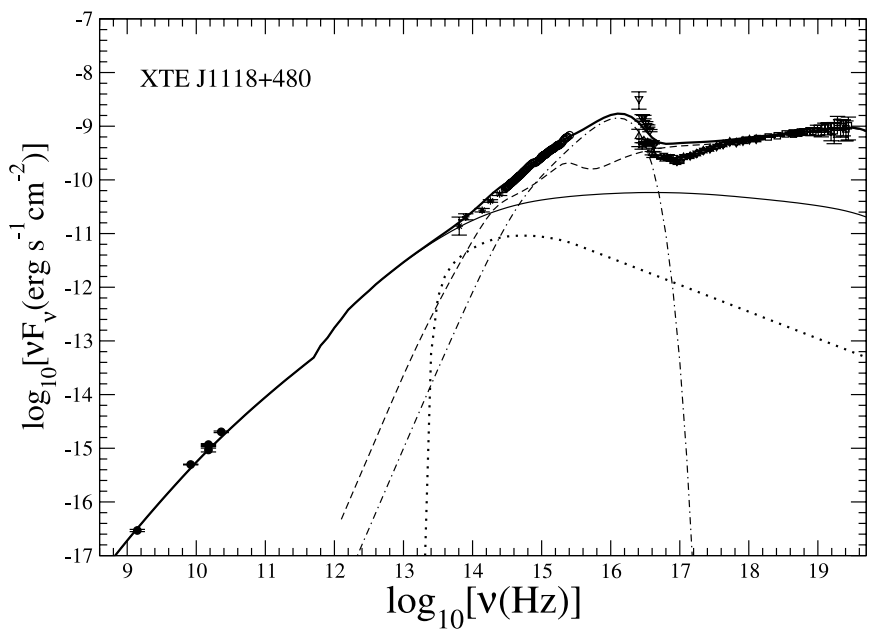

FIG. 2.-Accretion-jet model of XTE J1118+480. The dashed and dotdashed lines show the emission from the hot and cool accretion flows, respectively, as in Fig. 1. The thin solid line shows the emission from the jet. The sum of the three components, shown by the thick solid line, explains the spectrum all the way from radio to X-rays. The dotted line shows the synchrotron emission from power-law electrons that might be present in the hot accretion flow.

Narayan (1996) and Esin et al. (1997). They assume that the gas lost from the secondary initially forms a standard thin disk outside a transition radius $r_{\mathrm{tr}}$. At $r_{\mathrm{tr}}$, the cool disk is truncated and makes a transition to a hot accretion flow, described as an ADAF (Narayan \& Yi 1994, 1995b; Narayan et al. 1998b). E01 satisfactorily explain the X-ray, EUV, and UV spectra of the source, but their model slightly underpredicts the optical flux and significantly underpredicts the IR fluxes. They do not include radio measurements in their work, but it is quite clear that their model cannot account for the emission at radio wavelengths.

In contrast, Markoff et al. (2001) propose that the SED of XTE $\mathrm{J} 1118+480$ is dominated by synchrotron radiation from a jet, although they also need a truncated accretion disk to explain the UV and EUV spectra. Inside the truncation radius, they assume that the accretion flow becomes an ADAF-like accretion flow. However, unlike E01, they ignore the radiation from the ADAF.

No attempts have been made to explain the observed timing properties with either of the above models. Merloni et al. (2000) consider both spectral and timing data in their work, but their magnetic flare model predicts that the disk emission should peak at about $0.2 \mathrm{keV}$, which is in disagreement with the EUVE and Chandra data. In addition, the model implies almost no time lag between optical and X-ray photons, which seems to be at odds with the measurements. Recently, Malzac et al. (2004) have proposed a time-dependent, coupled disk-jet model for XTE J1118+480, which has some resemblance to the model we discuss in this paper. Whereas our model attempts to fit the spectral data (see the following sections), Malzac et al. concentrate on understanding the timing features. As they pointed out, because of the complexity of the time evolution of the accretionjet system, detailed modeling is impossible. They thus adopt a phenomenological approach. They model the variability by assuming random fluctuations of the output power from the disk and the jet, with the power being injected from a reservoir of stored magnetic field. By carefully choosing their parameters, they are able to reproduce almost all the observed timing features. These parameters can, in principle, constrain the dynamics and geometry of the accretion flow. One of their interesting results is that they can rule out models in which the energy budget is 
completely dominated by either the jet or the accretion flow; rather, they favor a model in which both components contribute.

In the present paper, we describe a coupled accretion-jet model to simultaneously account for both the spectral and timing properties of XTE J1118+480. We propose that the X-ray spectrum is produced mainly by the ADAF-like hot accretion flow, whereas the radiation at longer wavelengths comes from a jet (as in active galactic nuclei [AGNs]). A similar idea has been suggested previously (e.g., Hynes et al. 2000; McClintock et al. 2001b; Chaty et al. 2003). In $\S 2$ we describe the model and discuss how it can explain the SED of XTE J1118+480. In $\S 3$ we show that the observed temporal properties can also be accommodated qualitatively within the model. We conclude in $\S 4$ with a summary and discussion. We present in the Appendix technical details on calculating the jet emission.

\section{FITTING THE SPECTRUM}

\subsection{Accretion Flow}

The accretion component of our model is implemented in nearly the same manner as in E01, i.e., the accretion flow consists of an inner ADAF and an outer thin disk. However, we have taken into account advances in our understanding of the ADAF during the past 10 years. First, both numerical simulations (Stone et al. 1999; Hawley \& Balbus 2002; Igumenshchev et al. 2003) and analytical work (Narayan \& Yi 1994, 1995a; Blandford \& Begelman 1999; Narayan et al. 2000; Quataert \& Gruzinov 2000) indicate that probably only a fraction of the gas that is available at large radii actually accretes onto the black hole. The rest of the gas is either ejected from the flow or is prevented from being accreted by convective motions. The details are likely to depend on the accretion rate.

We note that the outflow (and convection) is ultimately the result of the accreting gas acquiring a positive Bernoulli parameter, as emphasized by Narayan \& Yi (1994, 1995a). Furthermore, the effect is strongest when the accretion rate is much below the threshold above which ADAF ceases to exist. Thus, accretion flows in highly underluminous sources, like Sgr A* or quiescent X-ray binaries, are expected to have strong outflows. On the other hand, the Bernoulli parameter decreases with increasing radiative efficiency and in fact becomes negative when the radiative efficiency is large enough. Therefore, for more luminous systems like XTE J1118+480 in outburst and other $\mathrm{X}$-ray binaries in the low/hard state, which have relatively high accretion rates and radiate fairly efficiently, we expect outflows and convection to be less well developed. In the present paper, we allow for this effect by adopting the following phenomenological prescription for the change in mass accretion rate as a function of radius. We assume that in the hot flow

$$
\frac{d \ln \dot{M}(r)}{d \ln r} \equiv s(r)
$$

where

$$
s(r)= \begin{cases}s_{0} f(r), & \text { if } 0 \leq f(r) \leq 1, \\ 0, & \text { if } f(r) \leq 0 .\end{cases}
$$

Here $s_{0}$ is a constant, which we set to $s_{0}=0.3$, as suggested by our previous modeling of the highly advection dominated source Sgr A* (Yuan et al. 2003). The parameter $f(r)$ is the advection factor of the accretion flow, defined as

$$
f(r) \equiv \frac{q_{\mathrm{adv}}}{q_{\mathrm{vis}}} \equiv \frac{q_{\mathrm{vis}}-q_{\mathrm{ie}}}{q_{\mathrm{vis}}},
$$

where $q_{\mathrm{adv}}, q_{\mathrm{vis}}$, and $q_{\mathrm{ie}}$ are the rates of energy advection, viscous heating, and Coulomb collision cooling for the ions, respectively. When the accretion rate is very low, as in the case of Sgr A*, $q_{\text {vis }} \gg q_{\text {ie }}$, so $f(r)=1$ and $s(r)=s_{0}$. In this case, from equation (1) we have the usual form, $\dot{M}=\dot{M}_{0}\left(r / r_{\text {tr }}\right)^{s_{0}}$, where $\dot{M}_{0}$ is the accretion rate at the transition radius $r_{\text {tr }}$ (or the outer boundary of the ADAF). We adopt $s_{0}=0.3$, as in the case of Sgr A*, because the physics of the outflow should be the same as long as $f(r)=1$, even though the accretion rates (in Eddington units) can be quite different. We should note, however, that our results are not sensitive to the exact value of $s_{0}$.

A negative value of $f$ in equation (2) means that advection plays a heating rather than a cooling role. In this case, the hot accretion flow is described by a luminous hot accretion flow (hereafter LHAF) model, which is a natural extension of an ADAF to higher accretion rates (Yuan 2001, 2003). From ADAF to LHAF, both $\dot{M}$ and the radiative efficiency increase continuously and smoothly. Yuan \& Zdziarski (2004) argue that for luminous X-ray sources, such as the low/hard states of some BHCs and Seyfert 1 galaxies, the luminosity may be above the highest luminosity an ADAF can reach but could be accommodated by an LHAF. We allow for an LHAF in this work, because it is unclear at present which solution, ADAF or LHAF, applies to XTE J1118+480. We simply refer to both the ADAF and LHAF solutions as "hot accretion flows."

We calculate the global solution of the hot accretion flow, starting at $r_{\text {tr }}$ and integrating inward. The numerical details can be found in Yuan (2001). One main difference with E01 is that we solve the radiation hydrodynamics equations selfconsistently, and thus we obtain the exact value of $f(r)$ at each radius. In contrast, E01 used the approximation that $f(r)$ has a constant average value at all radii. On the other hand, we treat Comptonization within a local approximation, whereas E01 computed the Comptonization globally using the method described in Narayan et al. (1997a).

The radiation processes we consider include bremsstrahlung, synchrotron emission, and the Comptonization of both synchrotron photons from the hot accretion flow and soft photons from the cool disk outside $r_{\text {tr }}$. The emission from the outer cool disk is modeled as a multicolor blackbody spectrum. The effective temperature as a function of radius is determined by the viscous dissipation and the irradiation of the disk by the inner hot flow.

Yuan \& Zdziarski (2004) found that to explain the X-ray emission of most black hole X-ray binaries, $\alpha \gtrsim 0.1$ is required (see also Narayan 1996). We fix $\alpha$ and the magnetic parameter $\beta$ (defined as the ratio of the gas pressure to the sum of gas and magnetic pressure) at their "typical" values, $\alpha=0.3$ and $\beta=$ 0.9 . We set $\delta=0.5$, i.e., $50 \%$ of the viscous dissipation heats electrons directly. The exact value of $\delta$ does not affect our results very much, since the $\dot{M}$ required to model XTE J1118+480 in outburst is relatively high, so the main heating mechanism for electrons is energy transfer from ions via Coulomb collisions. In this sense $\alpha, \beta$, and $\delta$ are not free parameters, although we should emphasize that large uncertainties exist here. We set the mass of the black hole at $M=8 M_{\odot}$, the distance to the source at $D=1.8 \mathrm{kpc}$, and the binary inclination $\theta=70^{\circ}$ (McClintock et al. 2001a; Wagner et al. 2001). Following E01, we estimate the outer radius of the cool disk using Paczyński's formula (Paczyński 1971): $r_{\text {out }}=3 \times 10^{4} r_{\mathrm{S}}\left(10 M_{\odot} / M\right)^{2 / 3}$, where $r_{\mathrm{S}} \equiv$ $2 G M / c^{2}$ is the Schwarzschild radius of the black hole. The free parameters of the accretion flow are the transition radius $r_{\text {tr }}$, the accretion rate at the transition radius $\dot{M}_{0}$, and an outer boundary condition, the temperature of the accretion flow at $r_{\text {tr }}$ (Yuan 1999). 


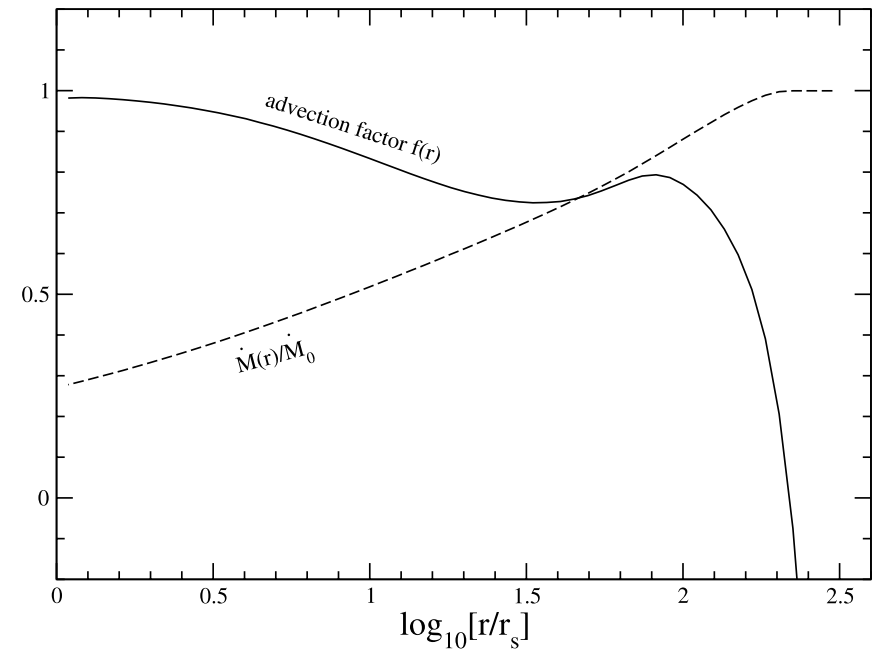

FIG. 3.-Advection factor $f$ (defined in eq. [3]) and the scaled mass accretion rate $\dot{M}(r) / \dot{M}_{0}$ as a function of radius for the hot accretion flow model shown in Fig. 1. Negative values of $f$ indicate that the accretion flow is in the LHAF regime rather than the ADAF regime at these radii. The solution is basically an ADAF.

Figure 1 shows the spectral fitting results obtained with the accretion flow model. The values of the parameters are $\dot{M}_{0}=$ $0.05 \dot{M}_{\text {Edd }}$ and $r_{\text {tr }}=300 r_{\mathrm{S}}$. The X-ray emission is produced by Comptonization in the hot flow. The main seed photons are from synchrotron emission by the thermal electrons in the hot flow (as assumed in the original ADAF model of Narayan \& Yi 1995b), as opposed to the blackbody emission of the thin disk. This is also consistent with the prediction of Wardzinski \& Zdziarski (2000), given that XTE J1118+480 is not very luminous. For more luminous sources, the seed photons may be dominated by blackbody emission from the thin disk. The EUV and UV emission in the model is mostly from the outer thin disk. The fit is satisfactory, although the optical fluxes are slightly underpredicted. The fact that the UV/optical emission is dominated by the thin disk explains the presence of Balmer jump absorption and emission lines and reprocessing features in the data $(\S 1)$. The IR and radio fluxes are significantly underpredicted, however (see Fig. 2). Figure 3 shows the profiles of the advection factor $f(r)$ and the fractional mass accretion rate $\dot{M}(r) / \dot{M}_{0}$ as a function of radius. We see that $f(r)$ is positive over much of the flow, except near $r_{\text {tr }}$. Since most of the radiation comes from the inner region, where $f(r)>0$, the solution is in the ADAF rather than LHAF regime, consistent with E01. This is because the luminosity of XTE J1118+480 is not high.

While our results are in general agreement with those of E01, there are two noteworthy differences. The first is that our value of $r_{\mathrm{tr}}\left(=300 r_{\mathrm{S}}\right)$ is significantly larger than that of E01 $\left(r_{\mathrm{tr}}=\right.$ $\left.55 r_{\mathrm{S}}\right)$. This discrepancy is mainly due to two reasons. First, E01 adopted a no-torque boundary condition at $r_{\mathrm{tr}}$, while we apply this condition at the marginally stable orbit of the black hole. Second, in E01 the mass accretion rate of the thin disk follows $\dot{M}(r)=\dot{M}_{0}\left(1-r_{\text {tr }} / r\right)$, while we simply use $\dot{M}(r)=\dot{M}_{0}$. Both differences are related to the physics of the transition of the accretion flow at $r_{\mathrm{tr}}$, which is highly uncertain at present, so it is not clear which approach is more appropriate. As a comparison, $r_{\mathrm{tr}}=352 r_{\mathrm{S}}$ in Chaty et al. (2003), who fitted the EUV spectrum, while $r_{\text {tr }}=17 r_{\mathrm{S}}$ in Frontera et al. $(2001,2003)$, who fitted the iron line and reflection features. The second difference between our model and E01 is that the value of $\dot{M}$ in E01 $\left(\dot{M}_{0}=\right.$ $\left.0.02 \dot{M}_{\text {Edd }}\right)$ is significantly smaller than ours $\left(\dot{M}_{0}=0.05 \dot{M}_{\text {Edd }}\right)$.
This is primarily because (1) we include an outflow in our calculations, so the accretion rate in the inner region is smaller than that at $r_{\text {tr }}$ (see Fig. 3, where $\dot{M} \sim 0.03 \dot{M}_{\text {Edd }}$ near the black hole in our model, close to E01's value), and (2) we use the pseudoNewtonian potential of Paczyński \& Wiita (1980), while E01 used the general relativistic solution of Popham \& Gammie (1998) in calculating the radial velocity of the accretion flow. As shown by Narayan et al. (1998a), the latter gives a higher luminosity for the same accretion rate.

To account for the underprediction of the IR and radio fluxes, we first consider the effect of nonthermal electrons in the hot accretion flow. Since the inflowing gas is collisionless, processes such as MHD turbulence, reconnection, and weak shocks can accelerate electrons and generate a nonthermal tail at high energies in the electron distribution function. Yuan et al. (2003) found that the radio spectrum of $\mathrm{Sgr} \mathrm{A}^{*}$, which was underpredicted by a pure ADAF model with only thermal electrons, can be explained if roughly $1 \%$ of the electron energy is in nonthermal electrons. We tested this idea for XTE J1118+480. The dotted line in Figure 2 shows the (absorbed) synchrotron emission from nonthermal electrons. We see that there is a sharp cutoff below about $10^{13} \mathrm{~Hz}$, so the emission from nonthermal electrons is unable to fit the radio and IR fluxes. This result is not sensitive to how much energy the nonthermal electrons have. In the case of Sgr A*, the emission from nonthermal electrons extends to a much lower frequency and forms a power-law spectrum. The difference between Sgr A* and XTE J1118+480 is that in the latter case the density is several orders of magnitude higher. Therefore, the magnetic field in XTE J1118+480 is much stronger, and the lowest frequency that the power-law electrons emit is much higher. We conclude that the accretion flow alone cannot account for the low-frequency spectrum of XTE J1118+ 480 at radio and IR wavelengths. Some other component, most likely a jet, is required.

\subsection{Coupled Accretion-Jet Model}

Jets are thought to occur in the low/hard state of BHCs (see Fender 2005 for a review). There have been many papers on the emission of radio jets in AGNs (e.g., Blandford \& Königl 1979; Ghisellini et al. 1985; Falcke 1996). In the present paper, following Spada et al. (2001), we adopt the internal shock scenario widely used in interpreting gamma-ray burst (GRB) afterglows (e.g., Piran 1999). The details of the model of the jet radiation are described in the Appendix. Briefly, we assume that near the black hole, a fraction of the accretion flow is transferred into the vertical direction to form a jet. Since the radial velocity of the accretion flow near the black hole is supersonic, a standing shock should occur at the bottom of the jet because of the bending. From the shock jump conditions, we calculate the properties of the postshock flow, such as the electron temperature $T_{e}$. We assume a constant $T_{e}$ in the jet, which is clearly oversimplified, since adiabatic expansion will cause the electrons to cool. However, this assumption has very little effect on the results, because the jet emission is dominated by the nonthermal electrons discussed below. We assume that the jet has a conical geometry with half-opening angle $\phi$ and that the bulk Lorentz factor of the jet $\Gamma_{j}$ is independent of distance from the black hole. We further assume that internal shocks occur as a result of the collision of shells with different $\Gamma_{j}$. These shocks accelerate a fraction of the electrons into a power-law energy distribution with index $p=2.24$ (e.g., Kirk et al. 2000). The steady state energy distribution of the accelerated electrons is carefully determined, since it is important for calculating the emitted spectrum. The effect of radiative cooling is considered 
in this process. Following the widely adopted approach in the study of GRBs, we specify the energy density of accelerated electrons and amplified magnetic field by two free parameters, $\epsilon_{e}$ and $\epsilon_{B}$. We then calculate the radiative transfer by both thermal and power-law electrons in the jet, although we find that the latter plays a dominant role. Only synchrotron emission is considered, since Compton scattering is not important in this case (see also Markoff et al. 2001).

The thin solid line in Figure 2 shows the emission of the jet. The parameters are the mass-loss rate in the jet $\dot{M}_{\text {jet }}=2.5 \times$ $10^{-4} \dot{M}_{\text {Edd }}$, which is about $0.5 \%$ of the accretion rate in the accretion disk; $\phi=0.1 ; \epsilon_{e}=0.06 ; \epsilon_{B}=0.02$; the bulk Lorentz factor of the jet $\Gamma_{j}=1.2$; and the length of the jet, $\sim 13 \mathrm{AU}$. The values of $\epsilon_{e}$ and $\epsilon_{B}$ are well within the typical range obtained in GRB afterglows (e.g., Panaitescu \& Kumar 2001, 2002), and the length of the jet is consistent with the observed upper limit of $65 D(\mathrm{kpc}) \mathrm{AU}$. The value of $\Gamma_{j}$ is well within the range obtained by combining observations and numerical simulations: $\Gamma_{j} \lesssim 1.67$ (Gallo et al. 2003).

We see from Figure 2 that the jet emission fits the lowfrequency radiation very well. The IR flux is dominated by the jet, while from optical to UV the jet becomes less important. The contribution of the jet to the EUV and X-rays is negligible. We should point out that the solution shown is not unique and that the jet parameters are not as well constrained as those of the accretion flow. However, the results are not very sensitive to the values of the jet parameters.

It is interesting to check whether a pure thermal jet can also explain the data. We find that we can get an equally good fit to the spectrum if we adjust the geometry and $T_{e}(z)$ profile of the jet carefully. In this model, we only need a tiny fraction of the gas in the accretion flow, $\sim 0.003 \%$, to go into the jet. However, the required temperature is very high, $T_{e} \sim 10^{10} \mathrm{~K}$. In addition, the jet velocity has to be very low, $\sim 100 \mathrm{~km} \mathrm{~s}^{-1}$; otherwise, the required magnetic field in the jet becomes unrealistically large. Such a low speed close to the black hole seems unphysical.

\section{INTERPRETING THE TIMING FEATURES}

\subsection{QPOs}

Numerous models have been proposed to explain the QPO phenomenon in X-ray binaries (see the review by van der Klis 2000). In some models, the QPO frequency is associated with the Keplerian frequency of the accretion flow at a special radius, the transition radius $r_{\text {tr }}$ in our case. For example, Giannios \& Spruit (2004; see also Rezzolla et al. 2003) suggest that the QPO can be excited by the interaction of the inner hot accretion flow and outer thin disk. The QPOs then result from the basic $p$-mode oscillations of the inner hot accretion flow with a frequency roughly equal to the Keplerian frequency at $r_{\text {tr }}$. The Keplerian frequency at $r_{\text {tr }}=300 r_{\mathrm{S}}$ is $\sim 0.22 \mathrm{~Hz}$, which is roughly consistent with the observed QPO frequency of $\sim 0.1 \mathrm{~Hz}$. Because the entire region of the hot flow oscillates collectively at the same frequency and the emission from the hot flow contributes somewhat at both the optical/UV and X-ray bands (see Fig. 1), the QPO should be observable at both optical/UV and X-ray wavelengths with the same frequency. Wood et al. (2000) find that the QPO frequency in XTE J1118+ 480 increases from 0.07 to $0.15 \mathrm{~Hz}$ during the outburst, while the $2-10 \mathrm{keV}$ X-ray flux slowly rises and then decreases. Our calculations do not show such a non-monotonic relationship, so the evolution of the QPO remains a puzzle. We should emphasize that the nonmonotonic change of the QPO frequency with the flux is not universal among BHCs. In fact, for most sources, the correlation seems to be monotonic (e.g., Cui et al. 1999).

\subsection{Variability Amplitude}

The variability amplitude from the jet is expected to be large, both from internal shocks and from possible instabilities in the jet. The hot accretion flow is thermally marginally unstable, so any perturbations in it will survive and move inward, as shown by numerical simulations (Manmoto et al. 1996) and analytical work (Yuan 2003). However, the growth timescale of the perturbations is longer than the accretion timescale, so the hot accretion flow is not threatened by the instability. The simulations further show that the simulated flux variation can account for the substantial variability observed in BHCs. On the other hand, the intrinsic variability of emission from the thin disk should be very weak, because the characteristic timescale is many hours even at $r_{\text {tr }}$, i.e., much longer than the observed approximately seconds or minutes variability timescale (e.g., Kanbach et al. 2001). The only source of variability of the thin disk emission is the reprocessing of the variable X-ray radiation, but the contribution of this component is very weak.

With the above knowledge, we can qualitatively understand variability amplitudes at different wavelengths. Large variability in the IR and X-ray bands is natural because the IR emission is dominated by the jet and the X-ray emission, by the hot flow. As the emission from the disk becomes more important in the optical and UV, the source varies less in these bands. The correlation between optical/UV and X-ray emission is easily understood, because the hot accretion flow contributes in both bands. H03 find that the SED of the variable component of the emission is roughly a power law, which they argued as being consistent with optically thin synchrotron radiation. However, given the fact that the rms amplitudes were derived from light curves with the same time resolution, it is actually not straightforward to interpret the result, since the intrinsic variability timescales at different wavelengths should be quite different. Moreover, the physical origin of the variability is likely to be complicated (e.g., Malzac et al. 2004). We note that a powerlaw SED of the variability does not arise naturally in a pure jet model (e.g., Markoff et al. 2001). For instance, if we assume that the variability is caused by fluctuations in $\dot{M}_{\text {jet }}$, such a model would predict a power-law index of 0.8 , which is the same as the X-ray spectral index, while the measured index of the variability spectrum is $\sim 0.59$ ( $\mathrm{H} 03$ ).

\subsection{Correlations between Optical/UV and X-Ray}

Suppose there is a perturbation due to an instantaneous increase of $\dot{M}_{0}$. The X-ray flux will increase. The increase in $\dot{M}$ will propagate inward with the accretion flow and eventually will lead to an increase in the mass-loss rate and thus the optical/UV emission from the jet. This could explain why the optical/UV variability lags the X-ray variability. Quantitatively, we find that in our model the optical/UV emission from the jet comes mainly from regions at a distance of about $d \sim$ $6000 r_{\mathrm{S}}$ from the black hole. This corresponds to a propagation time of $\sim d / c \sim 1.2 \mathrm{~s}$, consistent with the measured $\sim 1-2 \mathrm{~s}$ lag. The size of the optical emission region is $\sim 2 d \phi \sim 1200 r_{\mathrm{S}}$, where $\phi$ is the half-opening angle of the jet. The corresponding light crossing time is $1200 r_{\mathrm{S}} / c \approx 0.1 \mathrm{~s}$, consistent with the shortest variability timescale seen in the optical, $\sim 100 \mathrm{~ms}$ (e.g., Kanbach et al. 2001). Since the emission at longer wavelengths originates from regions farther away, the time lag should increase with increasing wavelength. 


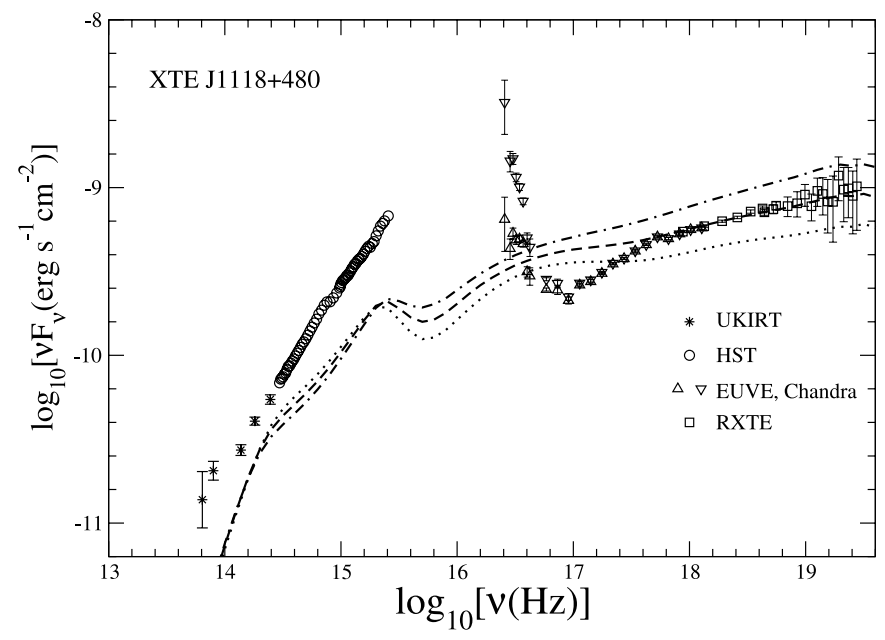

FIG. 4.-Model spectra from the hot accretion flow for three choices of $\dot{M}_{0} / \dot{M}_{\text {Edd }}: 0.04$ (dotted line), 0.05 (dashed line), and 0.06 (dot-dashed line). All other parameters are held fixed.

As for the negative lag, we note that for the parameters of our model (Fig. 1), an increase of $\dot{M}$ in the hot accretion flow results in a decrease of the optical/UV flux, as shown in Figure 4. The optical/UV emission from the hot accretion flow is mainly due to self-absorbed synchrotron emission, which depends on the profiles of $T_{e}$ and optical depth $\tau$. For our model, an increase in $\dot{M}$ causes a decrease in the flux. In our model the optical emission comes from $\sim 35 r_{\mathrm{S}}$, the UV from $\lesssim 10 r_{\mathrm{S}}$, and the X-rays from $\sim 7 r_{\mathrm{S}}-8 r_{\mathrm{S}}$. Thus, when $\dot{M}_{0}$ increases, the optical flux will first decrease, then the UV will decrease, and finally the X-ray flux will increase. This might be the origin of the negative lag of the optical/UV, as well as the negative correlation, and may also explain why the lag in the UV is shorter than in the optical. Since the emission from the hot accretion flow contributes less at shorter wavelengths in the optical/UV regime (see Fig. 1), we can also understand why the dip becomes weaker at shorter wavelengths. Since the IR flux from the hot accretion flow does not vary with varying $\dot{M}_{0}$ (see Fig. 4 ), we predict that such a negative lag should be absent between IR and X-ray emission.

Quantitatively, however, we are not able to account for the magnitude of the negative lags. The viscous timescale at $\sim 35 r_{\mathrm{S}}$ is $\sim 0.1 \mathrm{~s}$, which is more than 20 times smaller than the observed $2-5 \mathrm{~s}$ negative lag seen in the optical. This might be due to an approximation in the outer boundary condition we assume for the global solution. For technical reasons, we set the angular velocity of the flow at $r_{\text {tr }}$ to be substantially sub-Keplerian, $\Omega\left(r_{\text {tr }}\right) \sim$ $0.5 \Omega_{\mathrm{K}}$, even though it should be super-Keplerian (Abramowicz et al. 1998); otherwise, the viscous dissipation would be negative and the solution would be unphysical (see also Manmoto et al. 1997). Since the centrifugal force is the dominant factor determining the radial velocity of the accretion flow, our approximation makes the radial velocity much larger than it should actually be and thus leads to a shorter viscous timescale. In addition, the viscosity parameter $\alpha$ may be smaller than the value we adopted, which will again result in a longer viscous timescale.

Finally, we note that an increase of $\dot{M}$ in the cool thin disk will obviously result in an increase in the optical/UV emission. However, such an increase is unlikely to be seen in the crosscorrelation analysis, since the accretion timescale in the thin disk is on the order of hours.

\section{SUMMARY AND DISCUSSION}

The observational data on XTE $\mathrm{J} 1118+480$ are almost unique among all current BHCs. The spectral and timing information impose very strong constraints on theoretical models and provide us with an opportunity to understand in detail the inflow and outflow processes around black holes. In this paper we explain how these observations can be understood in the context of a coupled accretion-jet model. In our model, the accretion flow is described as a geometrically thin cool disk outside a transition radius $r_{\text {tr }}$ and a geometrically thick hot accretion flow inside $r_{\text {tr }}$, as in the model of E01. We adopt a phenomenological prescription for the magnitude of the mass outflow from the hot accretion flow (eqs. [1]-[3]). The free parameters describing the accretion flow are the transition radius $r_{\text {tr }}$, the mass accretion rate at $r_{\mathrm{tr}} \dot{M}_{0}$, and the outer boundary condition at $r_{\mathrm{tr}}$. The spectrum due to the accretion flow alone is shown in Figure 1. The $\mathrm{X}$-ray emission is dominated by Comptonization of synchrotron photons in the hot accretion flow, and both the EUV and UV are dominated by the cool disk. The fit is quite satisfactory in these bands. The optical flux is slightly underpredicted, however, and the IR and radio spectra are significantly underpredicted (Fig. 2). These results are very similar to those of E01.

Obviously, we require an additional component in the model to explain the IR and radio fluxes. We first consider the possibility of nonthermal electrons in the hot accretion flow, but we find that this idea does not work. We stress, however, that the failure does not mean that there are no nonthermal electrons in hot accretion flows. Such electrons might, for instance, be responsible for the hard tail in the spectrum of Cyg X-1 in the low/hard state (McConnell et al. 2000).

Having eliminated nonthermal electrons as an explanation for the low-frequency emission of XTE J1118+480, we argue that the radiation must originate in a jet. Assuming that a small fraction of the mass in the accretion flow is transferred to the jet, we calculate the jet emission using the internal shock scenario that is widely adopted in the study of GRB afterglows. The results of the accretion-jet model are shown in Figure 2. We find that the radiation from the jet can account for all of the radio and IR emission and part of the optical/UV emission. The required mass-loss rate in the jets is about $0.5 \%$ of the accreted matter.

The coupled accretion-jet model not only explains the spectrum, it also qualitatively explains many of the timing features observed in XTE $\mathrm{J} 1118+480$. These features include the frequency of the QPO; the similarity of the QPO frequency in the optical, UV, and X-ray bands ( $\S 3.1)$; the dependence of the variability amplitude on wavelength $(\S 3.2)$; and the positive and negative time lags between optical/UV and X-ray emission (§ 3.3). Quantitatively, however, we are not able to account for the magnitude of the negative time lag between X-ray and optical/UV emission $(\S 3.3)$.

It is interesting to examine the energetics of the accretion flow and the jet in our model. The total accretion power is $P_{\text {acc }}=\dot{M}_{0} c^{2} \sim 5 \times 10^{38} \mathrm{ergs}^{-1}$, and the power lost in the outflow is $P_{\text {outflow }} \equiv P_{\text {acc }}-\dot{M}\left(r_{\mathrm{S}}\right) c^{2}=3.6 \times 10^{38} \mathrm{ergs} \mathrm{s}^{-1}$. The X-ray luminosity emitted by the hot accretion flow is $L_{\mathrm{X}} \sim$ $2 \times 10^{36} \mathrm{ergs} \mathrm{s}^{-1}$, the optical/UV luminosity emitted by the thin disk is $\sim 2 \times 10^{36} \mathrm{ergs} \mathrm{s}^{-1}$, and the jet power is $P_{\text {jet }}=$ $\Gamma_{j}^{2} \dot{M}_{\mathrm{jet}} c^{2} \sim 3.6 \times 10^{36} \mathrm{ergs} \mathrm{s}{ }^{-1}$, which is $\sim 2$ times $L_{\mathrm{X}}$. For comparison, Malzac et al. (2004) require $P_{\text {jet }} / L_{X} \sim 10$ to reproduce the main timing features of XTE J1118+480, while Fender et al. (2001) estimate $P_{\text {jet }} / L_{\mathrm{X}} \gtrsim 0.2$. The luminosity emitted by the jet in our model is $L_{\text {jet }} \sim 2 \times 10^{35} \mathrm{ergs} \mathrm{s}^{-1}$, so the radiative efficiency of the jet is $\sim 0.055$, roughly consistent 
with the estimate of $\sim 0.05$ by Fender et al. (2001) but larger than the value of $\sim 0.003$ in Malzac et al. (2004). Thus, there are differences in both the value of $P_{\text {jet }} / L_{\mathrm{X}}$ and the efficiency of the jet between our model and that of Malzac et al. (2004). One reason for the discrepancy is that Malzac et al. assume the optical flux to be completely dominated by synchrotron emission from the jet, while our detailed modeling shows that the contribution from the accretion flow and the jet are comparable in the optical band (Fig. 2). Thus, more power from the jet is required in their model. In addition, the estimated value of $L_{\text {jet }}$ in Malzac et al. (2004) is only $5 \times 10^{34} \mathrm{ergs} \mathrm{s}^{-1}$, which is $\sim 4$ times smaller than ours. This is because they integrate the jet emission from radio to optical, while in our model the jet emission extends up to X-rays (see Fig. 2).

Assuming $P_{\text {acc }}-P_{\text {outflow }}=1.4 \times 10^{38} \mathrm{ergs} \mathrm{s}^{-1}$ to be the accretion power in the inner region of the accretion flow from which most of the X-ray and jet power originates, we see that only $L_{\mathrm{X}} /\left(P_{\text {acc }}-P_{\text {outflow }}\right) \sim 1 \%$ is released through the X-ray emission and $\sim 2 \%$ is channeled into the jet, while most of the accretion power is stored in the accretion flow and advected into the black hole. In other words, XTE J1118+480 is radiatively quite inefficient, in agreement with the conclusion of Malzac et al. (2004). The small ratio of the jet power to the accretion power also justifies our approximation that the jet has very little effect on the global solution of the hot accretion flow. We should point out that some uncertainties exist in the above estimations concerning the jet, since the jet parameters in our model are not as well constrained as the parameters of the accretion flow.

Several other caveats also need to be mentioned. First, we adopt a pseudo-Newtonian potential rather than the exact general relativistic approach when we calculate the dynamics of the hot accretion flow. Second, we adopt a sub-Keplerian angular velocity at the transition radius, whereas the rotation here should be super-Keplerian. The main effect of these two approximations is that the radial velocity in the hot flow is larger than it should actually be, and thus the density is smaller than the correct value. We believe that most of the effect is absorbed in the accretion rate parameter $\dot{M}_{0}$. However, the approximations do affect some quantitative results, such as the time lag between optical/UV and X-ray emission. Third, we have not explored fully the parameter space. The values of several parameters such as $\alpha, \beta$, and $\delta$ are fixed in our calculations (to $0.3,0.9$, and 0.5 , respectively). Investigating their effects in detail by surveying their entire parameter space would be very time consuming and is beyond the scope of the paper.

The philosophy of this paper is that the hard X-ray emission comes from the hot accretion flow via thermal Comptonization and that the contribution from the jet is negligible in this band. This is different from the model of Markoff et al. (2001), in which synchrotron radiation from the jet dominates in X-rays. We note that many details of the X-ray observations of BHCs have been successfully explained with a hot accretion flow model (see the review by Zdziarski \& Gierliński 2004), and it remains an open question whether the jet model can do equally well. Poutanen \& Zdziarski (2002) and Zdziarski et al. (2003) have pointed out some difficulties with the jet proposal. For example, the nonthermal synchrotron emission in this model cannot produce a sharp enough cutoff at high energies, and the predicted spectrum is not as hard as the spectra observed in many BHCs. In addition, the jet model should yield X-ray variability virtually independent of energy, which is in strong disagreement with the observational data. Finally, it is unclear whether the model can explain the various timing features of XTE J1118+480 described in this paper.

Of course, for some black hole sources the emission from the jet dominates over the accretion flow in the X-ray band. BL Lac objects are a well-known class in which this situation is known to exist. In previous work we have discussed this possibility also for two other sources, Sgr A* and NGC 4258 (Yuan et al. $2002 \mathrm{a}, 2002 \mathrm{~b}$ ). In the case of NGC 4258, the jet emission dominates the accretion flow because we require a significant fraction of the accretion flow to be transferred to the jet, $\dot{M}_{\text {jet }} / \dot{M}_{0} \approx$ $10 \%-25 \%$, which is more than $\sim 20$ times higher than in XTE $\mathrm{J} 1118+480$. Such a high value perhaps implies that the black hole in NGC 4258 is very rapidly spinning. In the case of Sgr A*, the value of $\dot{M}_{\text {jet }} / \dot{M}_{0}$ is similar to that in XTE J1118+480, but the $\mathrm{X}$-ray emission from the jet is comparable to that from the accretion flow. This is because the accretion rate (in Eddington units) in Sgr A* is much lower. The flux from the accretion flow, which comes from (multi-order scattering) Comptonization radiation, increases much faster with the accretion rate than that from the jet, which is from synchrotron and (one-order scattering) synchrotron self-Compton emission. Therefore, the ratio of jet to disk flux increases with decreasing Eddington-scaled accretion rate.

Recently, a very interesting correlation between radio and X-ray fluxes has been discovered in GX 339-4. The correlation extends over more than three decades in X-ray flux (Corbel et al. 2003). Such a correlation likely exists in other BHCs and even in AGNs (Gallo et al. 2003; Merloni et al. 2003; Falcke et al. 2004). The correlation is sometimes used as evidence for a jet origin for the X-ray emission of BHCs (e.g., Markoff et al. 2003). However, Heinz (2004; see also Merloni et al. 2003) recently pointed out that if the electron energy spectrum is not too steep and if radiative losses are included in determining the electron energy spectrum, both of which are required by observations, the jet model cannot explain the radio-X-ray correlation. Merloni et al. (2003) further showed that the X-ray emission is unlikely to be produced by radiatively efficient accretion (as in the sandwiched corona + disk geometry); rather, the accretion flow must be radiatively inefficient. Our preliminary investigations indicate that the radio-X-ray correlation can be explained in the context of our accretion-jet model (Yuan \& Cui 2004).

We thank S. Chaty, R. I. Hynes, and J. E. McClintock for providing us with the data. Helpful comments by S. Chaty and J. E. McClintock are acknowledged. This work was supported in part by NASA grants NAG5-9998 and NAG5-10780 and NSF grant AST-0307433.

\section{APPENDIX}

\section{THE INTERNAL SHOCK MODEL FOR JET RADIATION}

We adopt the internal shock scenario to calculate the emission from the jet, similar to Spada et al. (2001). We are interested only in the time-averaged spectrum. Following Blandford \& Königl (1979), we assume that the jet has a conical geometry, with a semiangle $\phi$ 
whose axis makes an angle $\theta$ with the direction of the observer. The jet has a constant velocity characterized by a bulk Lorentz factor of $\Gamma_{j}$ and has a constant plasma temperature. The mass-loss rate in the jet is

$$
\dot{M}_{\text {jet }}=\pi z^{2} \phi^{2} \rho(z) v_{j}
$$

The quantity $\rho(z)$ is the mass density of the jet plasma at distance $z$ from the black hole, measured in the jet comoving frame.

The main assumption in the internal shock scenario is that the central power engine produces energy that is channelled into jets in an intermittent way; thus, faster shells will catch up with slower ones, and internal shocks are formed in the jet. The minimum distance the shells propagate before collision occurs is $z_{0} \sim \Gamma_{j}^{2} r_{\mathrm{S}}$ (Piran 1999; Spada et al. 2001). Our results are not sensitive to its exact value.

The bulk Lorentz factor of steady jets in BHCs is likely to be only mildly relativistic (Fender 2005), e.g., $\Gamma_{j} \lesssim 1.67$ from Gallo et al. (2003). In this case, for an adiabatic index of $4 / 3$ the energy density of the internal shock is (Piran 1999)

$$
e_{2}=\gamma_{2} n_{2} m_{p} c^{2},
$$

where $\gamma_{2}=\left[\left(\Gamma_{j}^{2}+1\right) / 2\right]^{1 / 2}$ is the Lorentz factor of the formed internal shock and $n_{2}=\left(4 \gamma_{2}+3\right) n_{1}$ is the postshock number density, where $n_{1}$ is the preshock number density in the jet determined by equation (A1).

The shock will heat plasma in the jet, generate/amplify the magnetic field, and accelerate a small fraction of electrons into relativistic energy. We assume that the fraction of accelerated electrons in the shock is $\xi_{e}$ and fix $\xi_{e}=1 \%$. Given the uncertainty in shock physics, as is the usual approach, we introduce two dimensionless parameters, $\epsilon_{e}$ and $\epsilon_{B}$, which measure the fraction of the comoving internal energy of the internal shock stored in the accelerated electrons and magnetic field, respectively. Obviously, $\xi_{e}$ and $\epsilon_{e}$ are not independent.

Assume that the injected electrons after the shock acceleration have a power-law distribution with index $p$,

$$
n_{\mathrm{pl}}\left(\gamma_{e}\right) d \gamma_{e}=N_{\mathrm{pl}}(p-1) \gamma_{e}^{-p}, \quad \gamma_{\min } \leq \gamma_{e} \leq \gamma_{\max }
$$

We set $p=2.24$, according to the results of relativistic shock acceleration from Bednarz \& Ostrowski (1998) and Kirk et al. (2000). In this case $(p>2)$, we have

$$
N_{\mathrm{pl}}=\xi_{e} n_{2} \gamma_{\min }^{p-1}
$$

Now we calculate the value of $\gamma_{\min }$. We have

$$
N_{\mathrm{p} 1} m_{e} c^{2} \frac{p-1}{p-2} \gamma_{\min }^{2-p}=\epsilon_{e} U_{\mathrm{sh}}=\epsilon_{e}\left(\gamma_{2}-1\right) n_{2} m_{p} c^{2},
$$

where $U_{\mathrm{sh}}=\left(\gamma_{2}-1\right) n_{2} m_{p} c^{2}$ is the internal energy density of the internal shock. From the above equation and the definition of $\xi_{e}$, we can obtain

$$
\gamma_{\min }=\left(\gamma_{2}-1\right) \frac{p-2}{p-1} \frac{m_{p}}{m_{e}} \frac{\epsilon_{e}}{\xi_{e}} .
$$

The value of $\gamma_{\max }$ is not important if we are not interested in fitting the X-ray spectrum of XTE J1118+480 with jet emission. When radiative cooling of relativistic electrons is important, as in the present case of XTE J1118+480, the steady distribution of electrons is different from equation (A3). Defining a "cooling Lorentz factor" $\gamma_{c}$ at which the radiative timescale $t_{\text {rad }}$ is equal to the dynamical timescale $t_{\mathrm{dyn}}$ at distance $z$ in the jet,

$$
t_{\mathrm{rad}}=\frac{3}{4} \frac{8 \pi m_{e} c}{\sigma_{\mathrm{T}} \gamma_{c} \beta_{e}^{2} B^{2}}=t_{\mathrm{dyn}}=\frac{z}{c},
$$

depending on the relative values of $\gamma_{\min }$ and $\gamma_{c}$ there will be two cases for the steady distribution. When $\gamma_{\min }>\gamma_{c}$, we have

$$
n_{\mathrm{pl}}\left(\gamma_{e}\right) d \gamma_{e}= \begin{cases}N_{\mathrm{pl}}(p-1) \gamma_{c} \gamma_{\min }^{1-p} \gamma_{e}^{-2} d \gamma_{e}, & \gamma_{c} \leq \gamma_{e} \leq \gamma_{\min } \\ N_{\mathrm{pl}}(p-1) \gamma_{c} \gamma_{e}^{-(p+1)} d \gamma_{e}, & \gamma_{e} \geq \gamma_{\min } .\end{cases}
$$

When $\gamma_{\min }<\gamma_{c}$, we have

$$
n_{\mathrm{pl}}\left(\gamma_{e}\right) d \gamma_{e}= \begin{cases}N_{\mathrm{pl}}(p-1) \gamma_{e}^{-p} d \gamma_{e}, & \gamma_{\mathrm{min}} \leq \gamma_{e} \leq \gamma_{\mathrm{c}} \\ N_{\mathrm{pl}}(p-1) \gamma_{c} \gamma_{e}^{-(p+1)} d \gamma_{e}, & \gamma_{e} \geq \gamma_{\mathrm{c}}\end{cases}
$$

The magnetic field generated/amplified by the shock is determined by

$$
\frac{B^{2}}{8 \pi}=\epsilon_{B} U_{\mathrm{sh}}=\epsilon_{B}\left(\gamma_{2}-1\right) n_{2} m_{p} c^{2} .
$$


Since most of electrons may still be in a thermal distribution, we need to consider their role in emitting and absorbing photons. To this purpose, we need to know their temperature. One constraint comes from the following consideration. If the jet is formed at the innermost region of the accretion flow, within the sonic point at $\sim 10 r_{\mathrm{S}}$, since the accretion flow is supersonic, when it is bent into the vertical direction to form the jet a standing shock should occur. Note that the global solution of ADAF (e.g., Narayan et al. 1997b) does not find shocks. Our assumption of the bending shock is not in conflict with this result, since a jet was not considered in that calculation. On the other hand, a shock is found in the general relativistic MHD numerical simulations of jet formation (e.g., Koide et al. 2000). From the global solution of the accretion flow, we know the values of preshock quantities. Applying the shock jump conditions at the jet radius, we then are able to calculate the postshock quantities, including the electron temperature (see Yuan et al. 2002a for details). Adiabatic expansion will cause the electrons to cool, while the internal shocks in the jet will further heat the electrons. However, for simplicity, we do not consider these effects, since we find that the radiation from the power-law electrons dominates over that from thermal ones.

Now we are ready to calculate the emission from the jet. The emissivity from each location in the jet is

$$
I_{\nu}^{\mathrm{out}}(z)=\int_{0}^{\tau_{0}} e^{-\tau} S_{\nu}(\tau) d \tau \approx \frac{j_{\mathrm{th}}+j_{\mathrm{pl}}}{\alpha_{\mathrm{th}}+\alpha_{\mathrm{pl}}}\left(1-e^{-\tau_{0}}\right)
$$

where $\tau$ is the optical depth along the line of sight in the jet and $S_{\nu}=\left(j_{\mathrm{th}}+j_{\mathrm{pl}}\right) /\left(\alpha_{\mathrm{th}}+\alpha_{\mathrm{pl}}\right)$ is the source function, including the emission and absorption from both thermal $\left(j_{\mathrm{th}}, \alpha_{\mathrm{th}}\right)$ and power-law $\left(j_{\mathrm{pl}}, \alpha_{\mathrm{pl}}\right)$ electrons in the jet. We then integrate the emission from different distances in the jet to obtain the total emission. The relativistic effects are taken into account in the calculation. There is a remaining important point when we do the integration: we should not integrate all of the volume of the jet. A volume filling factor $f_{\mathrm{sh}}(<1)$ should be introduced. The value of $f_{\mathrm{sh}}$ is very uncertain. It obviously depends on the spatial density of the internal shocks in the jet. In addition, the generated/amplified magnetic field in the shock may survive for only a short time; this will further decease its value. We set $f_{\text {sh }}=0.1$ in our model. Fortunately, this value is not very important, since it can be absorbed in $\dot{M}_{\text {jet }}$.

\section{REFERENCES}

Abramowicz, M. A., Igumenshchev, I. V., \& Lasota, J.-P. 1998, MNRAS, 293, 443

Bednarz, J., \& Ostrowski, M. 1998, Phys. Rev. Lett., 80, 3911

Blandford, R. D., \& Begelman, M. C. 1999, MNRAS, 303, L1

Blandford, R. D., \& Königl, A. 1979, ApJ, 232, 34

Chaty, S., Haswell, C. A., Malzac, J., Hynes, R. I., Shrader, C. R., \& Cui, W. 2003, MNRAS, 346, 689

Corbel, S., Nowak, M. A., Fender, R. P., Tzioumis, A. K., \& Markoff, S. 2003, A\&A, 400, 1007

Cui, W., Zhang, S. N., Chen, W., \& Morgan, E. H. 1999, ApJ, 512, L43

Esin, A. A., McClintock, J. E., Drake, J. J., Garcia, M. R., Haswell, C. A., Hynes, R. I., \& Muno, M. P. 2001, ApJ, 555, 483 (E01)

Esin, A. A., McClintock, J. E., \& Narayan, R. 1997, ApJ, 489, 865

Falcke, H. 1996, ApJ, 464, L67

Falcke, H., Körding, E., \& Markoff, S. 2004, A\&A, 414, 895

Fender, R. P. 2005, in Compact Stellar X-Ray Sources, ed. W. H. G. Lewin \& M. van der Klis (Cambridge: Cambridge Univ. Press), in press (astro-ph/ 0303339)

Fender, R. P., et al. 2001, MNRAS, 322, L23

Frontera, F., et al. 2001, ApJ, 561, 1006 2003, ApJ, 592, 1110

Gallo, E., Fender, R. P., \& Pooley, G. G. 2003, MNRAS, 344, 60

Ghisellini, G., Maraschi, L., \& Treves, A. 1985, A\&A, 146, 204

Giannios, D., \& Spruit, H. C. 2004, A\&A, 427, 251

Haswell, C. A., Skillman, D., Patterson, J., Hynes, R. I., Cui, W., \& Chaty, S. 2000, IAU Circ., 7427, 1

Hawley, J. F., \& Balbus, S. A. 2002, ApJ, 573, 738

Heinz, S. 2004, MNRAS, 355, 835

Hynes, R. I., Mauche, C. W., Haswell, C. A., Shrader, C. R., Cui, W., \& Chaty, S. 2000, ApJ, 539, L37

Hynes, R. I., et al. 2003, MNRAS, 345, 292 (H03)

Igumenshchev, I. V., Narayan, R., \& Abramowicz, M. A. 2003, ApJ, 592, 1042

Kanbach, G., Straubmeier, C., Spruit, H. C., \& Belloni, T. 2001, Nature, 414, 180

Kirk, J. G., Guthmann, A. W., Gallant, Y. A., \& Achterberg, A. A. 2000, ApJ, 542,235

Koide, S., Meier, D. L., Shibata, K., \& Kudoh, T. 2000, ApJ, 536, 668

Malzac, J., Belloni, T., Spruit, H. C., \& Kanbach, G. 2003, A\&A, 407, 335

Malzac, J., Merloni, A., \& Fabian, A. 2004, MNRAS, 351, 253

Manmoto, T., Mineshige, S., \& Kusunose, M. 1997, ApJ, 489, 791

Manmoto, T., et al. 1996, ApJ, 464, L135

Markoff, S., Falcke, H., \& Fender, R. 2001, A\&A, 372, L25

Markoff, S., Nowak, M., Corbel, S., Fender, R., \& Falcke, H. 2003, A\&A, 397, 645

McClintock, J. E., Garcia, M. R., Caldwell, N., Falco, E. E., Garnavich, P. M., \& Zhao, P. 2001a, ApJ, 551, L147
McClintock, J. E., Narayan, R., \& Rybicki, G. B. 2004, ApJ, 615, 402

McClintock, J. E., \& Remillard, R. A. 2005, in Compact Stellar X-ray Sources, ed. W. H. G. Lewin \& M. van der Klis (Cambridge: Cambridge Univ. Press), chap. 4 , in press (astro-ph/0306213)

McClintock, J. E., et al. 2001b, ApJ, 555, 477

. 2003, ApJ, 593, 435

McConnell, M. L., et al. 2000, ApJ, 543, 928

Merloni, A., Di Matteo, T., \& Fabian, A. C. 2000, MNRAS, 318, L15

Merloni, A., Heinz, S., \& Di Matteo, T. 2003, MNRAS, 345, 1057

Narayan, R. 1996, ApJ, 462, 136

Narayan, R., Barret, D., \& McClintock, J. E. 1997a, ApJ, 482, 448

Narayan, R., Igumenshchev, I. V., \& Abramowicz, M. 2000, ApJ, 539, 798

Narayan, R., Kato, S., \& Honma, F. 1997b, ApJ, 476, 49

Narayan, R., Mahadevan, R., Grindlay, J. E., Popham, R., \& Gammie, C. 1998a, ApJ, 492, 554

Narayan, R., Mahadevan, R., \& Quataert, E. 1998b, in The Theory of Black Hole Accretion Discs, ed. M. A. Abramowicz, G. Bjornsson, \& J. E. Pringle (Cambridge: Cambridge Univ. Press), 148

Narayan, R., \& Yi, I. 1994, ApJ, 428, L13

. 1995a, ApJ, 444, 231 1995b, ApJ, 452, 710

Paczyński, B. 1971, ARA\&A, 9, 183

Paczyński, B., \& Wiita, P. J. 1980, A\&A, 88, 23

Panaitescu, A., \& Kumar, P. 2001, ApJ, 560, L49

. 2002, ApJ, 571, 779

Piran, T. 1999, Phys. Rep., 314, 575

Popham, R., \& Gammie, C. F. 1998, ApJ, 504, 419

Poutanen, J., \& Zdziarski, A. A. 2002, in New Views on Microquasars, 4th Microquasar Workshop, ed. Ph. Durouchoux, Y. Fuchs, \& J. Rodriguez (Kolkata: Center for Space Phys.), 87

Quataert, E., \& Gruzinov, A. 2000, ApJ, 539, 809

Remillard, R., Morgan, E., Smith, D., \& Smith, E. 2000, IAU Circ., 7389, 2

Revnivtsev, M., Sunyaev, R., \& Borozdin, K. 2000, A\&A, 361, L37

Rezzolla, L., Yoshida, S'i., Maccarone, T. J., \& Zanotti, O. 2003, MNRAS, 344, L37

Spada, M., Ghisellini, G., Lazzati, D., \& Celotti, A. 2001, MNRAS, 325, 1559

Spruit, H. C., \& Kanbach, G. 2002, A\&A, 391, 225

Stone, J., Pringle, J., \& Begelman, M. 1999, MNRAS, 310, 1002

van der Klis, M. 2000, ARA\&A, 38, 717

Wagner, R. M., Foltz, C. B., Shahbaz, T., Casares, J., Charles, P. A., Starrfield, S. G., \& Hewett, P. 2001, ApJ, 556, 42

Wardzinski, G., \& Zdziarski, A. A. 2000, MNRAS, 314, 183

Wood, K. S., et al. 2000, ApJ, 544, L45

Yuan, F. 1999, ApJ, 521, L55

2001, MNRAS, 324, 119 
Yuan, F. 2003, ApJ, 594, L99

Yuan, F., \& Cui, W. 2004, ApJ, submitted (astro-ph/0411770)

Yuan, F., Markoff, S., \& Falcke, H. 2002a, A\&A, 383, 854

Yuan, F., Markoff, S., Falcke, H., \& Biermann, P. L. 2002b, A\&A, 391, 139

Yuan, F., Quataert, E., \& Narayan, R. 2003, ApJ, 598, 301
Yuan, F., \& Zdziarski, A. 2004, MNRAS, 354, 953

Zdziarski, A. A., \& Gierliński, M. 2004, Prog. Theor. Phys. Suppl., 155, 99

Zdziarski, A. A., Lubinski, P., Gilfanov, M., \& Revnivtsev, M. 2003, MNRAS, 342,355 